\title{
Multiple renal tumorectomy in a Von Hipple Lindau patient. Combined retro/transperitoneal approach with intracorporeal hypotermia
}

\author{
Valentí Tubau ${ }^{1}$, Jose Luis Bauza ${ }^{1}$, Enrique Pieras ${ }^{1}$, Xavier Brugarolas ${ }^{1}$, Pedro Pizà ${ }^{1}$ \\ ${ }^{1}$ Department of Urology, Hospital Universitario Son Espases, Palma de Mallorca, Illes Balears, Spain
}

\begin{abstract}
Objective \& Introduction: To show the feasibility of a combined transperitoneal (TP) and retroperitoneal (RP) laparoscopic approach in a Von Hipple-Lindau (VHL) patient with multiple kidney tumors. VHL is an autosomal dominant inherited syndrome characterized by a high incidence of benign and malignant tumors and cysts in many organs. Renal cell carcinoma is one of the most common and a leading cause of mortality (1). Surgical approach is usually complex because of its multiplicity and the need of maximum kidney function preservation due to the risk of future recurrences $(2,3)$. Intracorporeal renal hypothermia may be useful in these cases to prevent permanent renal function loss (4).

Materials and Methods: A 40 years old male was being monitored for multiple bilateral renal masses. Family history included a VHL syndrome affecting his mother and sister.

Past medical history included a VHL syndrome with multiple cerebellar and medular hemangioblastomas, a pancreatic cystoadenoma and bilateral kidney tumors which had significantly grown up during follow-up.

The patient was scheduled for laparoscopic multiple partial nephrectomy. A combined TP and RP approach with intracorporeal hypothermia was chosen.

Results: A total of six right kidney tumors were removed. Operative time was $240 \mathrm{~min}$. Cold ischemia time was $50 \mathrm{~min}$. Average kidney temperature was $23.7^{\circ} \mathrm{C}$. Blood losses were negligible. The patient was discharged after 72 hours. No major changes in serum creatinine were found during the follow-up. Final pathology revealed a clear cell renal cell carcinoma, pT1a, ISUP grade 2 in most of the tumors but one ISUP grade 3. Surgical margins were negative.

Conclusions: Combined TP and RP is a feasible alternative for the treatment of multiple renal tumors. It's safe and effective, allowing the use of intracorporeal hypothermia which may improve postoperative renal function. Consistent experience is needed before embarking on this surgery.
\end{abstract}

\section{ABBREVIATIONS}

VHL $=$ Von Hipple-Lindau

$\mathrm{TP}=$ Transperitoneal

$\mathrm{RP}=$ Retroperitoneal

MRI = Magnetic Resonance Image

IVC = Inferior Vena Cava

MAL = Mid Axillary Line

ISUP = International Society of Urological Pathology

\section{CONFLICT OF INTEREST}

None declared. 


\section{ARTICLE INFO}

\section{Valentí Tubau}

https://orcid.org/0000-0002-8656-4784

Available at: http://www.intbrazjurol.com.br/video-section/20180803_Tubau_et_al

Int Braz J Urol. 2019; 45 (Video \#25): 1283-4

\section{REFERENCES}

1. Varshney N, Kebede AA, Owusu-Dapaah H, Lather J, Kaushik M, Bhullar JS. A Review of Von Hippel-Lindau Syndrome. J Kidney Cancer VHL. 2017;4:20-9.

2. Volpe A, Blute ML, Ficarra V, Gill IS, Kutikov A, Porpiglia F, et al. Renal Ischemia and Function After Partial Nephrectomy: A Collaborative Review of the Literature. Eur Urol. 2015;68:61-74.
3. Shekarriz B, Shah G, Upadhyay J. Impact of temporary hilar clamping during laparoscopic partial nephrectomy on postoperative renal function: a prospective study. J Urol. 2004:172:54-7.

4. Gill IS, Abreu SC, Desai MM, Steinberg AP, Ramani AP, Ng $C$, et al. Laparoscopic ice slush renal hypothermia for partial nephrectomy: the initial experience. J Urol. 2003;170:52-6.
Submitted for publication:

November 25, 2018

Accepted after revision:

March 23, 2019

Published as Ahead of Print:

May 15, 2019
Correspondence address:

Valentí Tubau, MD

Department of Urology

Hospital Universitario Son Espases

79 Valldemossa Rd

Palma de Mallorca, 70120, Spain

Telephone: +34 680 593-950

E-mail: valentitubau@gmail.com 